\title{
Cognition as shaking hands with the world. Introduction
}

\author{
Przemysław Nowakowski ${ }^{*}$ \& Tomasz Komendziński ${ }^{1}$ \\ Nicolaus Copernicus University \\ "pnowakowski[]avant.edu.pl \\ Received and accepted September 2014; published Autumn 2014.
}

Your fingers would remember their old strength better... if they grasped your sword.

Gandalf

(from the movie The Two Towers, second part of Lord of the Rings movie)

One of the most common questions in today's cognitive studies is the one regarding embodied cognition. The answer to this question draws our attention to many factors, including bodily actions, which also work to embody cognition. With this in mind, enactivism is included in discussions of embodiment. In the current issue we present texts in which a focus on enactivism itself is the leading topic.

If one were to describe declaratively the latest trend in cognitive studies, one would frequently refer to it as "embodied cognitive science"-sometimes with the addition of "radical embodied cognitive science"-or "enactive cognitive science". However, attempts at answering the question regarding the relations of range and meaning between these terms set in motion a never-ending discussion. The issues connected with embodied cognition and enactivism tend to refer back to areas outside the field of cognitive studies. Including the category of embodiment (often quite contingently connected with situated and distributed cognition) within the context of enactivism, whose methodological and historical-ideological status is ambiguous (is it a methodological approach? a trend?), generates additional problems and questions.

An attempt at introducing some order into the situation would require setting clear criteria and conducting a detailed notion analysis. One should always take into account various ways and contexts of using the categories of embodiment and enaction, which seem to lead us into the even broader waters of interdisciplinary studies. This is compounded by the additional problems

\footnotetext{
${ }^{1}$ Transl. Ewa Bodal.
} 
faced by the fields in which the aforementioned discussions are conductedincluding cognitive studies and interdisciplinary studies, and especially philosophy and psychology in particular. However, the present introduction does not aim at bringing order to these various levels on which enactive issues appear. Furthermore, there is also no guarantee that ordering is reasonable, necessary, or efficient for research, and whether this is even possible.

A certain common notional basis for enactivism is often pointed towards; it is comprised of such notions as autonomy, sense-making, structural coupling, self-organisation, agency, action, and sensorimotor dependencies. It is still a long way from showing the relations between these notions and from unifying enactivisms into one common theoretical proposal in a satisfactory manner. However, not only does this not stop the researchers, but it also encourages them to further-especially critical—studies, which will allow enactivism to discover itself anew.

At present, enactivism is explored in several partially different directions. From the radical biological roots of the theory of autopoiesis (Varela and Maturana), currently frequently referred to in the context of research on artificial life (Di Paolo, Froese), to research on cognition carried out through the sensorimotor system (Noë, O'Regan), to the role of interactions in social cognition and sense-making (de Jaeger, Gallagher), to seeking models of mind and the role of procedural knowledge in cognition (Hutto), a strong emphasis on the constructive character of cognition (Maturana) or pointing towards the key role of self-organisation and emotions in cognition (Ellis, Newton). The hereinabove distinguished notion basis refers precisely to all these issues. These remarks do not solve the issue of the variety of enactivism, but only point towards it.

"Enactivism" has its terminological source which is turning attention towards the role of very broadly understood actions as the key to understanding what cognition is. Shaun Gallagher (2013: 209) writes:

The enactive view of human cognition starts with the idea that we are action oriented. Our ability to make sense of the world comes from an active and pragmatic engagement with the world, along with our capacities to interact with other people.

McGann et al. (2013) are comparing cognition to a handshake and to dancing. They write that we have to use cognition when it is taking place, when it constitutes the action we are currently performing. This differentiates the enactivism from the concept of action. Much of research on action is-at least in light of some enactivist works-anti- or at least non-enactivist. Enactivism equates cognition with action, but it defines the criteria of "action" in its own way, focusing on its very performance. Other concepts focus on the mechanisms that make action possible. 
The enactivist, equating cognition with performing actions, finds the results in research on cognition being something akin to catching a flying ball. To put it differently-analogically from Gandalf's words to King Théoden-cognition happens when, in a specific context and with a specific tool (here: a sword), we start performing an action. This framing has both its advantages (e.g. observing cognition in statu nascendi may reveal many properties of the act of cognition), and disadvantages (it is possible that many of the processes that make acting possible do not reveal their basic properties only through action). Although the enactivist proposal seems very inspiring, is it enough for a revolution in, or a unification of, cognitive studies?

What seems to connect the enactivists, besides pointing towards action or towards a certain particular practice of cognition, is rejecting the existence of mental representations or their necessity for explaining the essence of cognition. However, the situation is more complex than it may initially seem. On the one hand, not all enactivists reject the concept of representations (e.g. Natika Newton), and on the other hand-a certain issue is located in the very status of representations criticised by enactivists, as well as the potential concepts of representation insusceptible to the critique of the enactivists (see: Steiner in the current issue). The same pertains to the notions we have listed above. Not every enactivist refers to such notions as self-organisation, autonomy, or sense-making, and the authors who do reach for them differ in their interpretations of these notions.

The present issue consists mainly of ten articles that are ten standpoints on enactivism: not only from the point of view of the critics (Aizawa, Steiner, Cummins, Bielecka) and the proponents (Gallagher \& Brower, Ellis, Li and Winchester), but also spokespeople for the moderate approach (Reid, Briscoe, Petit). Let us briefly outline the contents of the main part of the volume.

Aizawa (in this issue, as in: Aizawa 2014) presents doubts regarding enactivism, focusing on the category of "cognition", central to cognitive studies. Having shown why differentiating between cognition and behaviour is important for cognitive scientists, Aizawa analyses a number of works by enactivists in order to show that they frequently mistake cognition for behaviour. Steiner considers the enactivist critique of representationalism, showing that on the one hand, enactivists do not present a unified concept of representation in their critique, and, on the other hand, there are possibly such (nonreferential) concepts of representation that are immune to this critique. Bielecka proves that the radically externalist theory of content, as present in Manzotti's research, is not possible to support, pointing towards the doubtful concept of hallucinations by this author. Cummins criticises the basic notions of agency and autonomy, highlighting the fact that they are frequently mistaken by researchers. Briscoe, focuses on the spatial contents of experience and the meaning that "motor system" has for it, he presents Evans' (1982) con- 
cept as an alternative to Alva Noë's activism and proposes a concept that is more consistent with current empirical studies (while connecting Evans' concept with Millikan's (1984) research pertaining to mental representations). Petit develops his own interpretation of neurophenomenology (one of the elements of the enactivist programme), focusing on the brain mechanims of consciousness. Ellis also turns towards the functioning of the central nervous system, analysing the role of emotions, self-organisation and anticipation in consciousness, and using this point of view to criticise determinist and epiphenomenalist concepts of consciousness. Gallagher and Bower, while trying to make enactivism more embodied, point towards the important role of emotions and social factors; moreover (as with the two previous authors) they consider the way in which the functioning of the brain should be interpreted in the light of enactivism, especially in the context of research on predictive cognition. Some works consider the applied side of enactivism. The neurophenomenology mentioned above (see article by Petit) is one of the applications. Li and Winchester reflect on the concept of Freedom Education, showcasing both its advantages and its potential cohesion with the enactivist programme. In his interesting, historically grounded essay, Reid considers the relationship between enactivism and theories of teaching, as well as the potential pros and cons of enactivism in these theories, especially in concepts pertaining to teaching mathematics.

In a broader sense, the abovementioned articles can be significantly complemented by two interviews: one with Shaun Gallagher, and the other with Robert Rupert; they are able to direct the attention of the Readers towards more broadly conceived issues connected with situating cognitive processes.

This concludes a broad overview of the current issue. Before we invite you to read the articles collected herein, we will allow ourselves a few more remarks. We can sometimes notice certain continuities in the presented set of texts. One of the continued threads is the criticism of basic notions of enactivism. Aizawa ponders the direction for development and the character of the enactivist revolution. His attention focuses on whether the concept enactivists consider to be cognition should be called cognition at all. He claims that what we have to make do with here is something that was classically considered behaviour, and that makes the status of enactivism in cognitive studies especially interesting. According to the classical approach, it was behavior that was explained (explanandum), while the theories of cognition were supposed to explain this behaviour (they were explanantia). Enactivists-as Aizawa writes-on the one hand, consider cognition to be explanandum, and, on the other, reduce cognition to a form of behaviour. As a result, we have to make do with a particular, cognitive-enactive mixture of explananda and explanantia, declaratively incompatible, but at the same time, in fact and quite perversely compatible with classical cognitive science. Steiner critically presents the issue of relations between enactivism and representational concepts of 
cognition. In his critical reflections, Cummins reaches for the basic notions of the first wave of enactivist research. These works, together with Petit's and Bielecka's texts, point towards the weaknesses of enactivist proposals. As we believe, such approaches and their consequences are indispensable in order for enactivism to be able to develop fully and overcome the problems it encounters. Both internal and external criticism are not to be underestimated here ${ }^{2}$.

Gallagher and Bower-authors deriving from phenomenological backgrounds-consider the role of the nervous system in discussing enactivism in general. A similar path is pursued by Petit, who discusses one of the research programmes of enactivism: neurophenomenology, as well as by Ellis, who criticises the determinist concepts of consciousness. Such a strong interest in the central nervous system seems to be both surprising and valuable, as it makes possible the meeting of enactivism with the main trend of neurocognitive studies, as well as a reflection (in the light of these studies) on the participatory (Gallagher and Bower) and anticipating (Ellis) concepts of CUN, and also the role of time synchronizations in consciousness (Petit).

In the aforementioned articles we find references to Varela and Maturana's version of enactivism (Aizawa, Cummins, Steiner, Li \& Winchester, Ellis), as well as to the enactivist (or activist) proposals of Alva Noë (Briscoe), to Ellis and Newton's framing of enactivism (Ellis), enactivism as proposed by Daniel Hutto (Aizawa) or enactivism in robotics (Bielecka). It is, however, visible that the role of the beginnings of the idea of enactivism remains not to be underestimated, as although the works we have collected frequently refer to very advanced, recent studies, they do not—as we can see-disregard the roots that can undoubtedly be located in Varela and Maturana's research.

Referring back to the quote that opens the present introduction, we can say that thanks to the aforementioned articles we can, in a certain way, get a hold on enactivism and face up to it, and, as a consequence, also to the problem of cognition. We believe that these works can (and should) constitute an important voice in the dispute over what the essence of enactivism (and also enaction) is, and what role it should play in research on cognition. This seems to be suggested both by the critical and the favourable framings of enactivism.

\footnotetext{
${ }^{2}$ There have already appeared a number of voices criticising enactivism. In our opinion, among the most important of these is the review of Alva Noë's first book written by Ned Block (2005) a review that, among its other features, charged enactivists (here: Noë) with crypto-behavioralism, as well as mistaking causality for constitution. Additionally, there are interpretation problems concerning basic notions, such as sensomotorical contingencies. In one of her recent texts, Frederique de Vignemont attacks enactivism (which she equates with sensorimotor approaches, as Noë also did), arguing with the claim of the enactivists that every experience constitutes certain particular sensorimotor laws). According to this researcher, it is possible to separate such experiences which - as it seems - cannot be connected with any laws of this kind (see: de Vignemont 2011, 2014).
} 
We hope that the contents of this volume will bring us closer to explaining at least some doubts referenced here and to realising what constitutes cognition according to enactivists-although they themselves believe that it is something as dynamic, momentary, and unstable as handshaking.

\section{References}

Aizawa, K. 2014. What is this cognition that is supposed to be embodied? Philosophical Psychology (ahead-of-print): 1-21.

Block, N. 2005. Review of Alva Noë, 'Action in Perception'. Journal of Philosophy, 102: 259-272.

De Vignemont, F. 2011. A mosquito bite against the enactive approach to bodily experiences. Journal of Philosophy, 108 (4): 188-204.

De Vignemont, F. 2014. Acting for bodily awareness. L. Shapiro, ed. Routldege Handbook of embodied cognition. Routldege: 287-295.

Evans, G. 1982. The varieties of reference. J. McDowell, ed. Oxford: Clarendon Press.

Froese, T., McGann, M., Bigge, W., Spiers, A., \& Seth, A. K. 2012. The enactive torch: A new tool for the science of perception. Haptics, IEEE Transactions on, 5(4): 365 375.

Gallagher, S. 2013. The Enactive Hand. The Hand, an Organ of the Mind: What the Manual Tells the Mental. MIT Press: 209-225.

Millikan, R. G. 1984. Language, thought, and other biological categories: New foundations for realism. MIT Press. 